\title{
Efficacy of microwave ablation for the treatment of endometrial carcinoma: A report of 3 cases
}

\author{
KOHEI NAKAMURA ${ }^{1}$, KENTARO NAKAYAMA ${ }^{1}$, MASAKO ISHIKAWA ${ }^{1}$, HIROSHI KATAGIRI ${ }^{1}$, \\ TOMOKA ISHIBASHI $^{1}$, EMI SATO ${ }^{1}$, CHIKA AMANO $^{2}$ and SATORU KYO $^{1}$ \\ Departments of ${ }^{1}$ Obstetrics and Gynecology and ${ }^{2}$ Organ Pathology, \\ Shimane University School of Medicine, Izumo, Shimane 6938501, Japan
}

Received November 27, 2014; Accepted December 3, 2015

DOI: $10.3892 / \mathrm{ol} .2016 .4374$

\begin{abstract}
Microwave endometrial ablation (MEA) is effective for the emergency control of uterine hemorrhage. However, to the best of our knowledge, there has been only a single report of life-threatening hemorrhage induced by endometrial carcinoma that was treated with MEA. The present report evaluates the efficacy of MEA as an emergency therapeutic option for the control of bleeding due to advanced endometrial carcinoma and minimally-invasive, early-stage endometrial carcinoma in 3 patients. MEA was able to effectively control massive uterine bleeding due to endometrial carcinoma in 2 patients with advanced disease and was curative in a patient with minimally-invasive endometrial carcinoma. Given its safety, simplicity and effectiveness, MEA may be utilized for the emergency treatment of uterine bleeding in advanced endometrial carcinoma, and may be used as a curative treatment in early-stage endometrial carcinoma.
\end{abstract}

\section{Introduction}

Severe genital bleeding is one of the most frequently observed problems affecting women, and may have a profound effect on physical, social and emotional quality of life (1). There are a variety of causes of abnormal uterine bleeding, including leiomyoma, endometrial polyps, dysfunctional bleeding, endometrial malignancy, arteriovenous malformation, retained products of conception and gestational trophoblastic disease (1).

Microwave endometrial ablation (MEA), using a frequency of $2.45 \mathrm{GHz}$, was initially developed by Kanaoka et al (2) at Iseikai Hospital (Osaka, Japan) for the treatment of functional and organic menorrhagia. A previous study performed by the

Correspondence to: Dr Kentaro Nakayama, Department of Obstetrics and Gynecology, Shimane University School of Medicine, 89-1 Enya-cho, Izumo, Shimane 6938501, Japan

E-mail:kn88@med.shimane-u.ac.jp

Key words: microwave endometrial ablation, uterine bleeding, endometrioid carcinoma present research group indicated that MEA was useful for the control of menorrhagia and life-threatening uterine hemorrhage (3). However, MEA guidelines in Japan state that the treatment is not suitable for endometrial malignancy (4), and there have been no reports that have evaluated the effectiveness of MEA for the treatment of endometrial carcinoma. To the best of our knowledge, there is only a single previous report of MEA being used to treat abnormal uterine bleeding caused by endometrial carcinoma (5).

Currently, the typical treatment for endometrial carcinoma is laparotomy with total abdominal hysterectomy, with or without pelvic lymphadenectomy (6). The effectiveness of MEA as a treatment for endometrial carcinoma remains to be elucidated. In the present three cases, the efficacy of MEA as a therapeutic option, for the control of massive bleeding due to advanced and minimally-invasive endometrial carcinoma was evaluated.

\section{Case report}

Case 1. A 59-year-old woman (gravida 7, para 2) was referred to Shimane University School of Medicine (Izumo, Shimane) in December 2011 for investigation and treatment of endometrial carcinoma. The patient had a 10-year history of uterine leiomyoma and menorrhagia, but no other significant past medical or surgical history. When the patient began having a small amount of persistent abnormal vaginal bleeding, she was treated with ethinyl estradiol and norgestrel (detail unknown), however, the bleeding continued. An outpatient endometrial cytological examination was performed, which revealed hyperplasia, indicating a high possibility of endometrial carcinoma. The patient's general physical examination was unremarkable. Her hemoglobin level was low (7.8 g/dl; normal range, 11.4-14.6 g/dl), and cancer antigen 125 (CA125; $17 \mathrm{U} / \mathrm{ml}$; normal range, $<25 \mathrm{U} / \mathrm{ml}$ ) and carbohydrate antigen $19-9(25 \mathrm{U} / \mathrm{ml}$; normal range, $<37 \mathrm{U} / \mathrm{ml})$ levels were not elevated.

Transvaginal ultrasonography revealed a highly echoic lesion in the uterus, measuring $35 \times 23 \mathrm{~mm}$. Emergency magnetic resonance imaging (MRI) revealed an irregular and expanded endometrium and a uterine mass which measured 40 × $30 \mathrm{~mm}$. The image additionally indicated potential deep invasion of the myometrium. Endometrial curettage was attempted in order to obtain a histological diagnosis of endometrial carcinoma, but the patient began to bleed profusely. As it was not possible 
to achieve hemostasis, emergency treatment was indicated; however, radical open surgery was not advisable due to the small number of gynecological oncologists available locally. Therefore, MEA was used to control the heavy bleeding, prior to proceeding with pre-planned radical open surgery.

Active bleeding from the uterus persisted during MEA; however, the estimated intraoperative blood loss was low. The patient was transfused with 4 units of packed red blood cells, and did not experience any discomfort, significant bleeding or untoward side effects over the subsequent several days.

The pathological result of the endometrial curettage was grade 1 endometrial adenocarcinoma. Curative treatment was subsequently performed in the form of total abdominal hysterectomy, bilateral salpingo-oophorectomy and pelvic lymphadenectomy. The International Federation of Gynecology and Obstetrics (FIGO) stage was IIIcl (7), due to lymph node metastasis. The patient was treated with six 3 week cycles of paclitaxel $\left(175 \mathrm{mg} / \mathrm{m}^{2}\right)$ and carboplatin chemotherapy (area under the curve=5; dose, $700 \mathrm{mg}$ ), which was well-tolerated. However, the patient succumbed to disease following recurrence 1 year subsequent to MEA treatment.

Case 2. A 43-year-old nulliparous woman was emergently transported to Shimane University School of Medicine in February 2012, presenting with shock due to excessive menorrhagia. The patient had a 10-year history of a submucosal uterine leiomyoma with menorrhagia, and was known to be mentally disabled. The patient's general examination was normal. Her lowest hemoglobin level, prior to transportation to Shimane University School of Medicine, was $5.1 \mathrm{~g} / \mathrm{dl}$.

Emergency MRI revealed an expanded endometrium and a uterine mass, measuring $30 \times 30 \times 15$ mm (Fig. 1). The image additionally indicated potential endometrial carcinoma invading the myometrium. The patient remained in shock, and MEA was suggested in order to achieve hemostasis and to delay a decision on radical treatment.

The patient was transfused with 6 units of packed red blood cells during MEA, and did not experience any discomfort, significant bleeding or untoward side effects during the postoperative course.

The result of endometrial curettage performed immediately prior to MEA was grade 1 endometrial adenocarcinoma. The patient subsequently underwent total abdominal hysterectomy, bilateral salpingo-oophorectomy and pelvic lymphadenectomy. The tumor was classified as FIGO stage IA, due to myometrial invasion of less than half the myometrial depth and no lymph node metastases. The patient required no additional therapy and remains alive without recurrence.

Case 3. A 44-year-old woman (gravida 2, para 2) was referred to Shimane University School of Medicine in January 2013 with persistent abnormal vaginal bleeding and severe anemia. The patient had a history of menorrhagia, but no other significant medical or surgical history. Her general examination was normal. The patient's hemoglobin level was $6.2 \mathrm{~g} / \mathrm{dl}$ and her CA125 level was not elevated.

MRI revealed uterine adenomyosis, and it was suggested that this was the cause of the patient's menorrhagia. There were no observations to suggest malignancy, such as an expanded endometrium or a uterine mass.

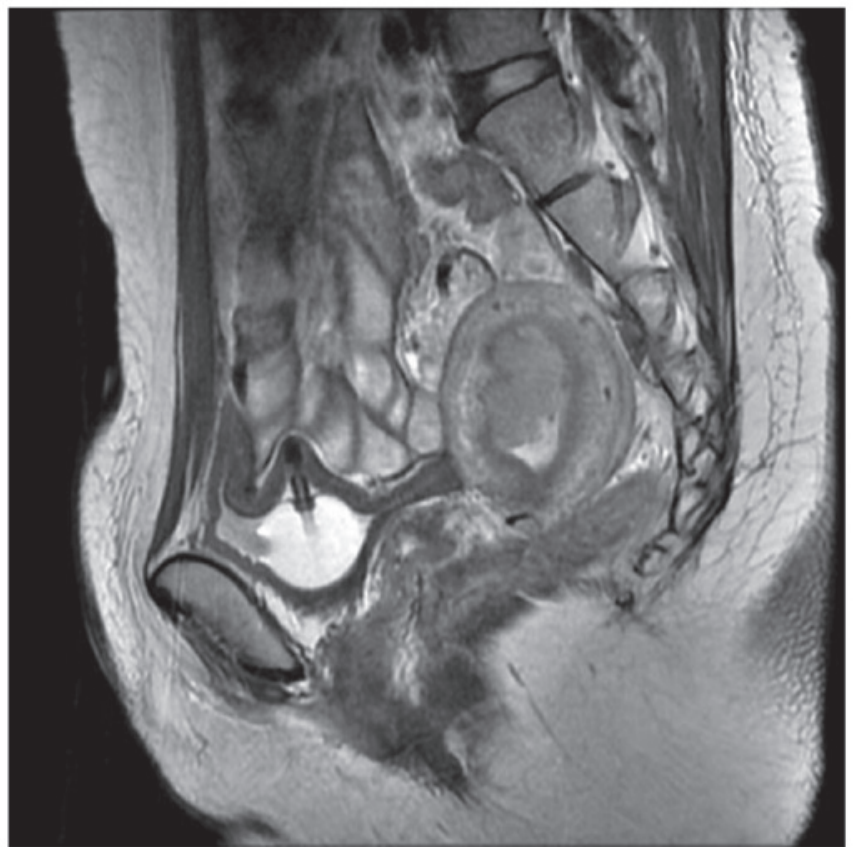

Figure 1. Magnetic resonance imaging of the pelvis. T2-weighted imaging revealed an expanded endometrium and a uterine mass measuring $30 \times 30 \times 15 \mathrm{~mm}$.
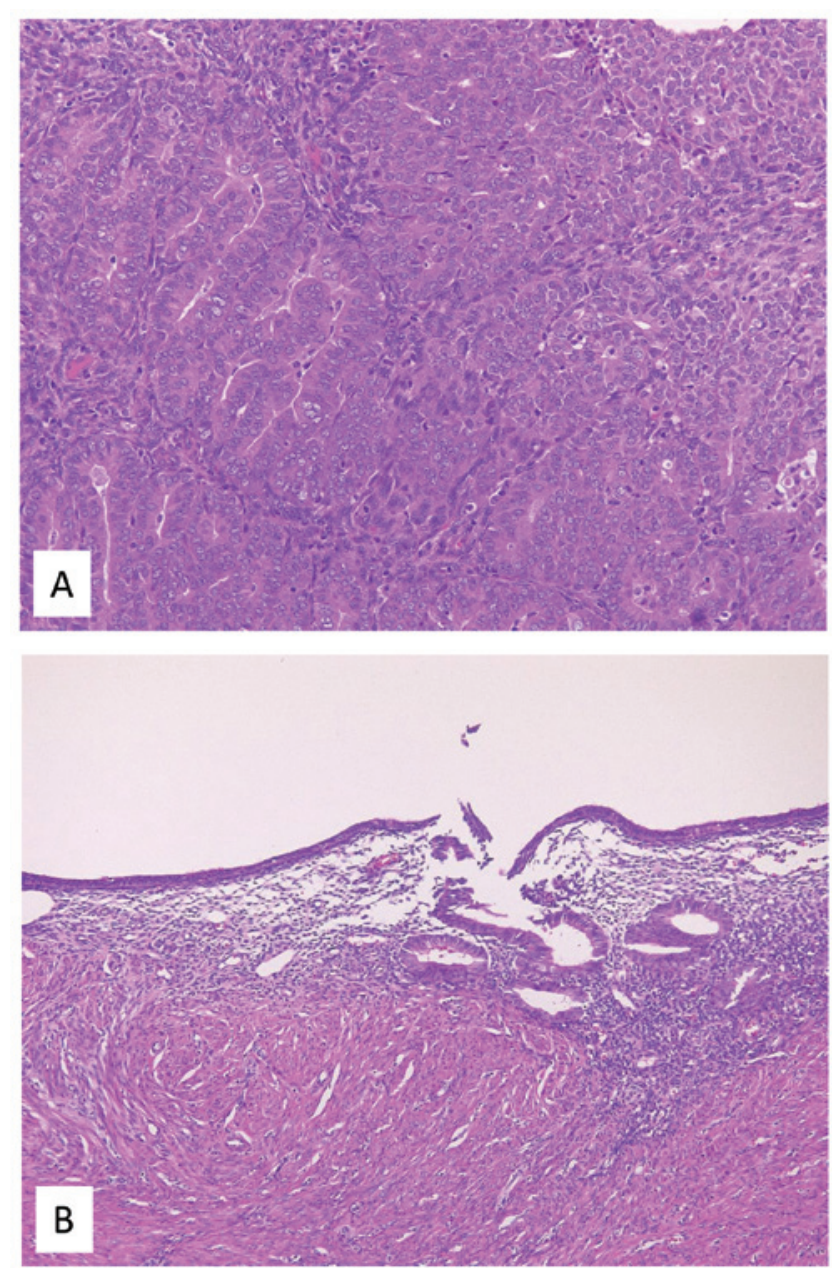

Figure 2. Histological examination. Sections were stained with hematoxylin and eosin. (A) Grade 1 endometrial endometrioid adenocarcinoma prior to MEA. (B) Uterine lining subsequent to MEA. The endometrium appeared completely normal. MEA, microwave endometrial ablation. 
MEA was performed, preceded by dilation and curettage for diagnosis and to rule out endometrial carcinoma. The patient demonstrated an unremarkable postoperative course and was discharged on the following day.

The result of the endometrial curettage was grade 2 endometrial endometrioid adenocarcinoma. The preoperative MRI had revealed no evidence of myometrial invasion; therefore, the malignant legion was hypothesized to be localized to the endometrium, and it was possible that the carcinoma was completely destroyed by the MEA. The patient was followed-up for 18 months, and no findings indicating a residual malignant lesion were identified by computed tomography (CT), positron emission tomography/CT or MRI examinations.

Although it appeared certain that this patient's carcinoma had been cured by MEA, the risk of recurrence was discussed. Ultimately, a decision to perform definitive treatment was reached. The patient underwent total laparoscopic hysterectomy with bilateral salpingo-oophorectomy. Pathological examination revealed no evidence of residual carcinoma (Fig. 2A and B). The patient remains alive without recurrence.

\section{Discussion}

The present study group previously reported that MEA is highly effective for the emergency treatment of life-threatening uterine hemorrhage $(3,8)$. Prior to the approval of MEA for insurance coverage in Japan, the present institution obtained approval to perform the procedure from the ethics committee of Shimane University Medical Department (Izumo, Japan), and this committee additionally approved the treatment for the patients in the present case series. Following comprehensive explanation of the risks and benefits of MEA, all patients provided written informed consent.

MEA was performed under spinal anesthesia using a device consisting of a sounding applicator and a microwave generator. The $2.45 \mathrm{GHz}$ microwaves were supplied by the Microtaze AZM-520 generator (Alfresa Pharma Corp., Osaka, Japan). Microwaves were transmitted at $70 \mathrm{~W}$ for $\sim 50 \mathrm{sec}$. In order to avoid perforating the uterus, transabdominal ultrasonographic guidance was used.

MEA guidelines state that suspicion of endometrial malignancy is a contraindication for the use of MEA (4); however, there is no clear evidence against the use of the procedure in these situations. To the best of our knowledge, MEA has been used only once in an attempt to treat endometrial carcinoma-associated uterine bleeding (5). In that case report, the authors suggested that MEA may be considered a palliative treatment for elderly women with early endometrial carcinoma who have severe complications and are therefore unfit for standard treatment (5). In the current case series, emergency control of uterine hemorrhage caused by endometrial carcinoma in two patients was achieved using MEA. In these patients, elective surgical treatment was subsequently successfully performed.

The MEA procedure is rapid and demonstrates a low level of blood loss compared with open surgery. The present experiences with endometrial carcinoma patients demonstrate that MEA may be used for emergency palliative control of uterine bleeding. However, it should be noted that the MEA procedure cannot be used for curative treatment in patients with advanced-stage endometrial carcinoma (4). Therefore, it is recommended that MEA be used for emergency control of life-threatening uterine hemorrhage, with subsequent laparotomy for the radical treatment of endometrial carcinoma.

In addition, a patient with endometrial carcinoma that was undiagnosed at the time of the procedure was treated with MEA. At the time of hysterectomy, 18 months subsequent to MEA, there was no malignancy detected following pathological examination. This suggests that malignant lesions localized to the endometrium may be treated using MEA.

In conclusion, the current case series concerning 3 patients suggested that MEA may be used to control life-threatening hemorrhage due to advanced-stage endometrial carcinoma, and may additionally be used to treat endometrial carcinoma localized to the endometrium. Future studies of additional cases are required to confirm the results of the present case series.

\section{References}

1. Cowan BD and Morrison JC: Management of abnormal genital bleeding in girls and women. N Engl J Med 324: 1710-1715, 1991.

2. Kanaoka Y, Hirai K and Ishiko O: Microwave endometrial ablation for an enlarged uterus. Arch Gynecol Obstet 269: 30-32, 2003.

3. Nakayama K, Rahman MT, Rahman M, Ishikawa M, Yeasmin S, Katagiri A, Iida K, Nakayama N, Aoki S and Miyazaki K: Microwave endometrial ablation is a highly efficacious way to emergently control life-threatening uterine hemorrhage. Arch Gynecol Obstet 283: 1065-1068, 2011.

4. Kanaoka Y, Ishikawa N, Asakawa Y and Nakayama K: Practice Guideline of MEA 2012. www.alfresa-pharma.co.jp/microtaze/MEAguideline2012.pdf (In Japanese). Accessed February 4, 2016.

5. Sharp N, Ellard M, Hirschowitz L, Malthouse S and Johnson N: Successful microwave ablation of endometrial carcinoma. BJOG 109: 1410-1412, 2002.

6. He H, Zeng D, Ou H, Tang Y, Li J and Zhong H: Laparoscopic treatment of endometrial cancer: systematic review. J Minim Invasive Gynecol 20: 413-423, 2013.

7. $\mathrm{Lu} \mathrm{Z}$ and Chen J: Introduction of WHO classification of tumours of female reproductive organs, fourth edition. Zhonghua Bing Li Xue Za Zhi 42: 649-650 (In Chinese).

8. Nakayama K, Ishibashi T, Ishikawa M, Katagiri A, Katagiri H, Iida K, Nakayama N and Miyazaki K: Microwave endometrial ablation at a frequency of $2.45 \mathrm{GHz}$ for menorrhagia: Analysis of treatment results at a single facility. J Obstet Gynecol Res 40: 224-229, 2014. 